\title{
Priority setting in health care systems: too much or not enough
}

\author{
Franz Porzsolt
}

Received: 1 February 2010 /Accepted: 1 February 2010 /Published online: 23 February 2010

(C) Springer-Verlag 2010

The more strategies someone recommends for setting priorities in health care, the more friends will be lost. Unfortunately, we ought to have both for a pleasant life, friends and recommendations for priority setting. This complicated solution may be achieved if we avoid teaching with our own recommendations, but rather identify and present the proposals of others, and exactly this is done in the current volume of the Journal of Public Health.

To categorize the accepted contributions, we differentiate two types of contributions: articles that complain of 'too much' of something and articles that express that there might be 'not enough' of something. As expected, there are more authors who complain about 'not enough' than authors who report about 'too much.'

A German-Austrian group analyzes part of our health care reforms and concludes that 'too much' planning of medical care generates more harm than good (Fülöp et al). 'Too much' confusion about the factors that influence the psychological distress among relatives among patients with chronic functional psychoses can be avoided (Nitsche et al.) by a careful analysis of suspected areas. 'Too much' is also a problem concerning allergic diseases in a rural area of Iran; whether it is too much dust or pollen or something else is a rather important question (Fereidouni). Definitely 'too much' is the use of cell phones while driving and the resulting crashes. All of us will be curious to see if we can

F. Porzsolt $(\bowtie)$

Clinical economics, University of Ulm,

Frauensteige 6,

89075 Ulm, Germany

e-mail: franz.porzsolt@uniklinik-ulm.de change the behavior of the drivers faster than the industry can develop cell phones that recognize risky situations and send a signal to the driver and insurance company. The study of an international group (Bener et al.) may well trigger some insurance companies to sponsor a new generation of cell phones.

'Not enough' attention is paid to culturally sensitive and gender-specific interventions for an etnically diverse adolescent population with overweight in California (Wilkosz et al.). A similar problem was addressed in Germany by Dreas and Hassel. They recognized that the transparency and information provided in 78 obesity prevention projects for kindergarden children were 'not enough' to draw meaningful conclusions. The new Master of Public Health curriculum for students in Serbia demonstrates that what has been done so far is 'not enough' to inform and educate the students and to support the development of democracy and welfare in Serbia (Jancovic et al.). At the managerial level the need for change is perceived, but in general there is 'not enough' understanding of what should be changed. Doctoral programs, lifelong learning programs and a larger research base in public health and management may improve the situation (Bjegovic et al.). Cho and Khang demonstrated in their Korean study that the Family Affluence Scale (FAS) can be used more frequently for studies in adolescents in Europe. Kuitto et al. concluded in their study that the uptake rates for primary and secondary prevention measures against cervical cancer were 'not enough' and could be enhanced. The reason why especially the elderly should be encouraged is not really clear. A convincing proposal is made by the Greifswald group (Hanan et al.). Microfinance is offered 'not enough' as a tool to finance medical devices, e.g., wheelchairs for disabled people, to generate income. This may be a more 
promising approach than many other activities that involve huge amounts of resources. We should also consider that it is 'not enough' to improve structures and processes unless the outcomes will be improved. Improved outcomes require the clear definition of goals. If we integrate economic principles in health care we should keep in mind the final goal of our actions (Porzsolt).
Finally, it is interesting to note that almost all of the described 'not enough' scenarios can effectively be supported just by providing high quality information. High quality information-i.e., information that is valid, important and applicable to our actual problems - seems to be one of the most valuable goods in all areas of health care. Let's go for it. 